\title{
Addressing Schistosomiasis in a Community in Nigeria: A Theoretical Approach
}

\author{
Anyanti J* (iD, Akuiyibo S (D, Onuoha $O$, Nwokolo E, Atagame $K$ and Braide EI
}

Society for Family Health, Abuja, Nigeria

*Corresponding author: Jennifer Anyanti, Society for Family Health, Abuja, Nigeria

\begin{abstract}
Schistosomiasis is one of several Neglected Tropical Diseases considered a major public health problem that affects tropical and sub-tropical countries. Residents of Gwako, a rural community in Nigeria's Federal Capital Territory (FCT) lack access to potable water supply with community members resorting to a dirty stream - an unhygienic water source. It is estimated that significant proportions or all Gwako residents are infested with schistosomiasis with very high transmission rates. Residents live with the disease and seem to have no agency or control over their health. Our research therefore sought to understand the community's perception, attitudes, and knowledge (KAP) as regards schistosomiasis prevention and control. The theory of reasoned action and of social diffusion were used to develop a conceptual framework and analyze findings of the research. The theoretical models also informed the development of an approach to intervene in Gwako to address schistosomiasis and other neglected tropical illnesses. Society for Family Health with funding from Procter \& Gamble and Global Citizens implemented a Water Sanitation and Hygiene (WASH) intervention in Gwako following the KAP research. Community residents showed high perception of the disease, substantial disease knowledge but poor knowledge of treatment and prevention. Findings from the study were used to develop an intervention to address the challenge with significant impact. The intervention which promoted the application of behavior change theories helped to reduce the feelings of helplessness among Gwako residents on schistosomiasis and helped to draw government support and action.
\end{abstract}

\section{Introduction}

Schistosomiasis is one of several Neglected Tropical Diseases (NTDs) considered major public health problems that affect tropical and sub-tropical countries such as Nigeria, Ghana, and Cameroon. The disease is endemic in several communities in Nigeria, such as Gwako in the Federal Capital Territory (FCT), Abuja. The disease causes economic and health inconveniences for patients and communities where it is endemic, and Nigeria is the most schistosomiasis-endemic African nation [1].

The country has an overall prevalence of $9.5 \%$ with about 24 million persons at risk of schistosomiasis [2]. The Federal Capital Territory where Gwako is located has a reported prevalence of urinary schistosomiasis of $31.30 \%$ [3]. In general, urinary schistosomiasis is a persistent health problem among children in schistosomiasis endemic communities. Poverty is a major driver facilitating the spread of schistosomiasis in Nigeria. Additionally, the lack of access to safe water and adequate sanitation and toilet facilities exacerbates the situation [4]. In endemic communities like Gwako, a large proportion of the population use unsafe streams, ponds and other seasonal stagnant water bodies for domestic and agricultural purposes [5]. The indiscriminate disposal of human faeces into water sources through open defecation also aids the spread of disease agents and vectors [4].

Gwako is a community in Gwagwalada Area Council in the Federal Capital Territory, Abuja. It has a population of about 50,000 persons with a third being under the age of five years old. Despite proximity to the $\mathrm{Ni}$ gerian Capital City Abuja, Gwako has very poor water, sanitation, and hygiene conditions, including very poor access to clean water. The community lacks several basic amenities, such as clean potable water, and private and public toilets. The major source of water for domestic and animal use is a river that is infested with disease vectors. This river serves as a source of water for both

Citation: Anyanti J, Akuiyibo S, Onuoha O, Nwokolo E, Atagame K, et al. (2021) Addressing Schistosomiasis in a Community in Nigeria: A Theoretical Approach. Int J Trop Dis 4:044. doi.org/10.23937/2643461X/1710044

Accepted: January 09, 2021; Published: January 11, 2021

Copyright: (C) 2021 Anyanti J, et al. This is an open-access article distributed under the terms of the Creative Commons Attribution License, which permits unrestricted use, distribution, and reproduction in any medium, provided the original author and source are credited. 
human and livestock use. Open defecation is a common practice in the community, and this is washed into the river when rain falls.

Children and adults get infected through bathing with water fetched from infested streams, and even from making visits to the infested streams to fetch water, washing or swimming in such waters [6]. Evidence from various studies in Nigeria has shown that communities and individuals are often unaware of the risks of transmission of schistosomiasis via cercariae-infested water bodies and hence more infections occur [7]. This challenge is concentrated in rural areas of the country, as levels of education are low and while many understand the likely links between infected waters and the illness, they are unable to implement practices that will improve knowledge, attitude and practices in the management of the disease [8]. Thus, despite perceived susceptibility to the disease, lack of autonomy of the people leads to the inability to implement preventive measures.

\section{Objective}

This study focuses on generating evidence for $\mathrm{Ne}$ glected Tropical Diseases (NTD) programming in Gwako community where NTDs such as schistosomiasis are endemic. It generates data that will help inform and guide behavior change programming by identifying community and individual experiences, perceptions and behavior change influences for the design and implementation of tailored interventions.

The research specifically provides information about:

- The community/individual experiences and perceptions about safe water and good environmental practices.

- The community/personal norms and values specifically the issues (influencing) their behavior towards prevention and care seeking for schistosomiasis and uptake of safe water and good environmental practices.

- The community/individual experiences and perceptions about schistosomiasis.

\section{Methodology}

A qualitative study was conducted amongst people who have lived in the community for at least a decade. This generated insights to help inform and guide behavior change programming by identifying community and individual experiences, perceptions and behavior change influences for the design and implementation of tailored interventions.

The method of data collection was Focus Group Discussions (FGDs) with various respondents selected from the community using discussion guides. The use of discussion guides gave flexibility to gain deep insights into knowledge, perceptions and behaviors of respondents.
Verbal informed consents were sought and received from all participants.

\section{Focus group discussions}

Focus group discussions (FGDs) were held with men and women in Gwako. These FGDs provided information about the community as well as personal norms and values, respondents insights around schistosomiasis and specifically the key determinants of preventive and care seeking behaviors for schistosomiasis. Of interest was whether there were differences between knowledge and perceptions of schistosomiasis between males and females, and between Gwari indigenes and non-indigenes groups. Topic guides were developed for each of the components of the FGDs using constructs from the Health Belief Model (HBM). Four focus groups were conducted among members of the community two male and two female groups, respectively. The 4 groups consisted of one male and one female Gbari indigenes group and one male and one female non-indigenes groups among those who have resided in the community for between 2 years to above 2 decades, making a total of 4 groups. The age range of FGD participants was between 18 and 48-years-old and the moderators ensured that differences between groups would be adequately elicited during the FGDs in a congenial atmosphere.

The FGDs had an average of 12 participants, and a total number of 48 randomly recruited participants were in the FGDs for the exercise - 24 males and 24 females. The participants were recruited randomly by visiting every $5^{\text {th }}$ house within the community and screening for eligibility using a screening questionnaire till the required number of participants were reached. Discussions were held in Hausa language by trained qualitative research facilitators.

\section{Ethical consideration}

This study was implemented following ethical standards in research. Ethical approval was received from the Gwagwalada Area Council Research Ethics Committee (REC) in the FCT. All persons implementing this study complied with all policies and procedures of the REC. Also, research participants were informed of all risks and protections in the oral assent/consent script. No participants were interviewed without their informed consent.

\section{Results}

\section{Socio-demographics of the respondents}

In total, 48 persons (24 males and 24 females each) aged $18-48$ years participated in the FGDs. There were two groups of 18 to 25-year-olds and 26 to 45 -year-olds for each gender: Making a total of four (4) groups of 12 persons each as shown in the Table 1. 
Table 1: FGD group composition.

\begin{tabular}{|l|l|l|l|}
\hline \multirow{2}{*}{ Gender } & \multicolumn{2}{|l|}{ Age Group } & Total \\
\cline { 2 - 3 } & $\mathbf{1 8 - 2 5}$ years & $\mathbf{2 6 - 4 5}$ years & \\
\hline Male & 12 & 12 & 24 \\
\hline Female & 12 & 12 & 24 \\
\hline Total & & & 48 \\
\hline
\end{tabular}

\section{Knowledge and awareness of schistosomiasis}

In general, there were high levels of disease awareness and disease prevalence shown among the community residents. Respondents were all able to spontaneously mention a number of diseases and ailments incidents in their environment which include: Malaria, Ringworm, Typhoid, Rashes, Measles, Dysentery, Stooling with mucus, High Blood Pressure, Ulcer and Body Itching, etc.

Schistosomiasis was mentioned by majority to be a very common disease in Gwako, affecting everyone including children and adults. The disease is locally called 'Pisarin jini' and its symptoms are said to be: Passing out urine mixed with blood, itching, vomiting, stooling with bloodstain, loss of weight, abdominal pain, body weakness, etc.

Community and individual experiences: All participants were aware of the disease and majority have experienced it at some point. They noted the disease is common and both the young and the old suffer from it. Few among the male non-indigene participants unaided mentioned 'Schistosomiasis' disease. It is called "pisarin jini" in Hausa which means "urinating blood".

More than half of the male/female respondents confirmed that they are currently suffering from the disease and were willing to urinate for signs of proof. In their own words:

"Many people suffered from it, especially children".

"Yes. Plenty for here, even me [pass stool] like that (with blood stains)".

"Yes,... children/adult [pass] urine and stool is stained with blood".

"It affected me. When I want to urinate, I feel pain and the blood stains my trousers".

"I have a sister that is experiencing the disease now".

"I suffer from the disease and I have a friend who is currently urinating with blood".

"One of my son is still stooling blood and [there is mucus in the stool]".

Perceptions about schistosomiasis transmission, prevention, treatment and control: The people associate the disease with the community river, some also think it is spiritual in nature, others say it is sexually transmitted. In their own words,

"That river is dirty, and it causes it, children are going there bathing/swimming from that water, they get that disease from that place".

"Some adults contract it through sexual intercourse"

"..... [it is from the] river water, when you take the river water you have the disease, the food and the chemical [in the water] that they are using are bringing us different kind of diseases".

"Through the contaminated water, people defecate everywhere, people fetch from the river water, drink with it, cook with it, there is no toilet in Gwako most of them run to the bush to toilet".

"Bad water, drinking of dirty water, no mosquito nets, lack of environmental sanitation, bathing with water without soak away when people cross it, you can contract it".

"We lack water. They drink, wash plate, cloth and swim/bath from the river, this causes it".

Respondents stated that the disease is communicable from person-to-person because almost all their family members contract it from sharing common household utilities. Another male indigene participant stated that three (3) members of his household were infected. In their words, the disease can be passed from one person to another:

"when you share the same toilet [improperly] used by an infected person".

"when you pass the same place that has been urinated on unconsciously by infected person, it is transferred from when an infected person [urinates] on the road, another person can contract it".

"By sharing the same food, water, and room with infected person".

"when an infected person urinates and someone go to the same place to urinate, the person will become infected as I was infected when my brother who was infected [and I] shared the same room".

"Open defecation by infected person and rainfall takes the [faecal] material to the river".

This long-dated disease in the community is perceived to be treatable although, it reoccurs because no preventive measures are available. Both modern and traditional medicines are used by respondents and community members for the treatment of this disease. "Sariki Bokah", a traditional healer in the community, when spoken with, emphasized the commonness and severity of the disease and that many people receive treatment from him. In the words of other respondents:

"It is treated with Indocin tabs and hot, when we put two capsule of Indocin with [alcohol] it healed us and 
the disease is completely cured".

"It can be treated with local herbs, those who can afford it go to the hospital".

"My neighbour whose child is urinating with blood, a praziquantel tablet was given. There are some drugs here that are used for it".

"I take my [child] to [patent medicine shop] and drug was purchased, [the] urination with blood has stopped while stooling is still happening".

"purchase drugs prescribed by people from local clinics which doesn't work but I received drugs when they were sharing it in the primary and junior secondary schools [in the community]".

Most times, respondents experience re-occurrence of schistosomiasis after treatment and sometimes it does not re-occur. Reoccurrence often depends on the kind of treatment received. In respondents' words:

"It reoccurs after two days or one week".

"Yes, one of my friend experienced reoccurrence".

"After treatment, it comes back". (unanimously agreed)

"Yes, there is reoccurrence as my sister was treated but up till now, it still exists".

"All respondents experienced re-occurrence".

"No reoccurrence in my neighbour son case".

In terms of preventive measures put in place against schistosomiasis, most respondents had this to say:

"Stop the children from going to the stream"

"Stop children from defecating openly by getting [toilets] for the children".

"Stop them from drinking, washing and swimming in the river".

"Having alternative basic amenities like good roads, bridges, water, electricity and re-orientating our children about going to the river to bath drink the dirty river water".

"We don't have any method to take because the river water is the only source of water for us".

"We should stop passing the river to the farm if government build a bridge across the river".

"No social amenities like toilet, good water and good environment, if they are not around we cannot prevent $i t^{\prime \prime}$.

"To prevent it we must keep good environment and not allow [the community] to be dirty".

Most individual members perceived their own ability to take action to address the challenge of schistosomiasis especially as regards hygiene and health seeking behaviors as low. They mentioned adoption of good hygiene practices as a way to prevent infection with the disease.

Schistosomiasis is perceived as a very serious illness by almost every household in the community, they know it is preventable and curable. However, the community feels overwhelmed and incapable of addressing the challenges and hope in God and the government for help. In their own words,

"We worry about it because of our children as our health center is not equipped. The cost and stress of treating the disease is a big challenge".

"It is a great problem beyond every individual and the community ... the government should look into alleviating or immunizing people against it... government should provide a better hospital in the community with good drugs for treatment when it occurs".

This endemic disease is a significant concern to Gwako community as people are afraid that if not addressed, it could soon lead to several deaths. Therefore, members of the community asked that the Nigerian Government come to their aid to provide clean potable water (borehole and pipe-borne water), public toilets, resuscitate the only primary health center in the community via renovation, staffing and provision of drugs. They also suggested that alternative amenities such as bridges should also be built across the river to avoid entry into the river with bare legs. Local and foreign health NGOs are also expected to implement interventions in the community that will help alleviate the sufferings of the people. In their words,

"Government should help with drugs as they cannot treat without drugs and not only drugs, but they should also equip the health centre, and bring water along with drugs to the health centre".

"Government should help bring pump water and borehole".

"Construct bridge across the river for us, government should be concerned with building bridges across the river so that we will stop crossing the river [on foot]".

"Government should introduce immunization against the disease".

"Government should go around to campaign to create awareness about prevention"

\section{Discussion}

The relatively high prevalence of Schistosomiasis in rural locations such as Gwako remains a public health concern in the eradication of NTDs in Nigeria, as there is limited access to quality healthcare services and social amenities in such locations. As observed in this study, the respondents had a good knowledge of the symptoms of Schistosomiasis. This finding is not unexpected 
since most of them have had the disease at one point in time or the other in their lifetime. A study conducted in rural Nigerian communities reported that almost four out of ten respondents had a history of Schistosomiasis infection [9].

Despite the widespread awareness of Schistosomiasis observed among the respondents in this study, certain misconceptions still exist among them regarding the mode of transmission of the causative agents of the disease. Spiritual causes were attributed to Schistosomiasis transmission among some of the respondents. Studies have shown that spirituality influences perception of health and disease in traditional African societies $[10,11]$. Also, sexual intercourse was identified as a mode of transmission of transmission in this study. Although there is evidence that the causative agent of urogenital schistosomiasis can travel into seminal vesicles, sexual transmission has not been reported [12].

Risky health behaviors are often difficult to change because they are mostly long-standing habits. Lack of opportunity or motivation sometimes hinders the adoption of healthy behaviors [13]. In this study, it was observed that although the respondents were aware of good hygiene practices as a means of prevention of schistosomiasis infection, they continued to engage in risky practices such as drinking and use of contaminated water for domestic purposes. Lack of access to good water supply encouraged the practice of consuming untreated water.

In response to this identified gap, the interventions introduced in this study were premised on the understanding that positive attitude and positive personal norms result in an individual's greater perceived control and increases the likelihood of intentions that govern improved changes in behavior, and that the use of positive role models would engender positive behaviors within the community. Strategic advocacy engagements with government, a community empowerment and a health promotion initiative were used to encourage good hygiene practices such as use of point-of-use water treatment solutions.

\section{Theoretical framework for selected intervention}

To understand the schistosomiasis situation in Gwako, the study team must comprehend the drivers of uptake of safe water, hygiene and clean environmental practices which are behavior change interventions. This study therefore also explored linkages in theories of Diffusion of Innovations (Dol) and Reasoned Action. These theories discuss dynamics of knowledge, risk perception and behavior-change in individuals [14]. Theory of reasoned action posits that positive attitude and positive personal norms results in an individual's greater perceived control and increases the likelihood of the person's intentions that govern changes in behaviour [15]. The theory of social diffusion posits that the use of positive role models would motivate positive behavior change within the community. These theories guided design and application of tools that elicited responses to gain understanding of the individual and the community in relation to these dynamics. The findings were used to design and implement interventions tailored to address the schistosomiasis endemic in Gwako.

\section{The intervention}

SFH's intervention applied key elements based on the above mentioned two theories. The interventions aimed to create positive attitudes and positive personal norms among community members and institutions regarding control and eradication of schistosomiasis. The traditional institution and community governance structures command influence and are respected in the community. In the same vein, community members trust the advice from health personnel at the PHC and some persons of influence.

The intervention included:

- Strategic advocacy engagements with government.

- A community empowerment and a health promotion initiative designed to address key identified issues from the research and community engagement. These were implemented through existing community structures including

- Traditional institutions,

- The ward development committee,

- The primary health care centre in the community, and

- Trained community health promoters.

- Distribution of products such as point-of-use water treatment solutions to engender behavior change to households.

\section{Intervention outcomes}

Through sharing stories of impacted persons and positive modelling of appropriate behavior by role models, the intervention:

- Empowered the community to appreciate their role in the control and eradication of schistosomiasis.

- Brought to the attention of government, local and international partners key WASH challenges, highlighting the impact of poor access to WASH on the individual, the community, and assisted community leaders and programmers in the identification of short, medium- and long-term solutions.

- Prodded positive actions by government. The FCT government tested samples from the communi- 
ty and planned a mass house to house treatment campaign of persons in the community.

Subsequently, evidence from the primary health centre in the community, showed a significant drop in hospital visits resulting from diarrhoeal disease. Following advocacy efforts and the showcasing of the schistosomiasis challenge in Gwako, the FCT health secretariat commenced sample collection and analysis and trained additional community health workers in water, sanitation and hygiene programming. A mass schistosomiasis treatment campaign of all households in Gawko was also scheduled.

\section{Conclusion}

Use of theoretical frameworks to analyze community perceptions of neglected tropical diseases and to also design community approaches are important in addressing NTDs in Africa. The urgent need to draw attention to the increasing NTD challenges such as schistosomiasis in communities is critical and will elicit government, community, and partners' response in addressing this neglected health area through community targeted initiatives.

Similarly, the application of appropriate behavior change theories in the design of interventions can be useful to identify key influencers and empower community action. This helps to reduce the feelings of helplessness communities feel around addressing NTDs. Community influencers and structures can be used to mobilize communities to act, to address issues, to the extent of drawing on government support and action. External media attention can also be drawn to the issue of NTDs using social diffusion (positive modelling, use of community networks and spreading the word through media).

\section{Authors Contribution}

JA conceptualised the study. OO and KA implemented the study, and $O O$ and DA implemented the intervention. JA and SA develop the first draft of the manuscript for the study, EN and EIB supported the preparation of the manuscript and the intervention. Meanwhile, all authors had input into manuscript development, review, and finalisation.

\section{Acknowledgements}

The authors wish to acknowledge the health care workers, community interpersonal communication agents and traditional and cultural leaders of Gwako community for their support and openness during the implementation of this research. We hope that this will contribute to bringing the desired change in the area of addressing NTDs in Gwako and similar communities in Nigeria.

\section{References}

1. Chitsulo L, Engels D, Montresor A, Savioli L (2000) The global status of schistosomiasis and its control. Acta trop 77: 41-51.

2. Global Network Neglected Tropical diseases (2015) Government of Nigeria releases new data on the prevalence of schistosomiasis and intestinal worms. SABIN: Vaccine Institute, USA.

3. Ifeanyi CIC, Matur BM, Ikeneche NF (2009) Urinary schistosomiasis and concomitant bacteriuria in the Federal Capital Territory Abuja Nigeria. New York Sci J 2: 1-8.

4. Bishop HG (2017) Menace of schistosomiasis: Its true neglected nature in Nigeria. MOJ Public Health 6: 421-426.

5. Accessing portable water in FCT rural communities. Daily Trust.

6. Ekpo UF, Laja-Deile A, Oluwole AS, Sam-Wobo SO, Mafiana CF (2010) Urinary schistosomiasis among preschool children in a rural community near Abeokuta, Nigeria. Parasit Vectors 3: 58.

7. Bishop HG, Inabo HI, Ella EE (2016) Prevalence and intensity of urinary schistosomiasis and their effects on packed cell volume of pupils in Jaba LGA, Nigeria. Edorium J Microbiol 2: 13-26.

8. Dawaki S, Al-Mekhlafi HM, Ithoi I, Ibrahim J, Abdulsalam AM, et al. (2015) The menace of schistosomiasis in Nigeria: Knowledge, attitude, and practices regarding schistosomiasis among rural communities in Kano State. PLoS One 10: e0143667.

9. Dawaki S, Al-Mekhlafi HM, Ithoi I, Ibrahim J, Abdulsalam AM, et al. (2016) Prevalence and risk factors of schistosomiasis among Hausa communities in Kano State, Nigeria. Rev Inst Med Trop São Paulo 58: 54.

10. Arrey AE, Bilsen J, Lacor P, Deschepper R (2016) Spirituality/religiosity: A cultural and psychological resource among Sub-Saharan African migrant women with HIVIAIDS in Belgium. PloS One 11: e0159488.

11. Asare M, Danquah SA (2017) The African Belief system and the patient's choice of treatment from existing health models: The Case of Ghana. Acta Psychopathologica 3: 49.

12. Crespillo-Andujar C, Díaz-Menéndez $M$, Mora-Rillo $M$ (2018) Evidence for previously unidentified sexual transmission of protozoan parasites. Emerg Infect Dis 24: 602603.

13. Fathnezhad-Kazemi A, Hajian S (2019) Factors influencing the adoption of health promoting behaviors in overweight pregnant women: A qualitative study. BMC Pregnancy and Childbirth 19: 43.

14. Sheeran P, Harris PR, Epton T (2014) Does heightening risk appraisals change people's intentions and behavior? A meta-analysis of experimental studies. Psychol Bull 140: 511-543.

15. Hale JL, Householder BJ, Greene KL (2002) The theory of reasoned action. In: The persuasion handbook: Developments in theory and practice. 259-286.

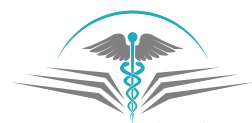

